\title{
Adult community-acquired pneumonia with unusually enlarged mediastinal lymph nodes: A case report
}

\author{
LANHUA ZHANG, SHIXIONG QIU, CUI TANG and JINMING XU \\ Department of Radiology, Yangpu Hospital, Tongji University School of Medicine, Shanghai 200090, P.R. China
}

Received October 13, 2015; Accepted February 14, 2017

DOI: $10.3892 /$ etm.2017.4449

\begin{abstract}
Adult community-acquired pneumonia (ACAP) is the most prevalent pulmonary infectious disease that may be asymptomatic or have varying clinical presentations. Patients with ACAP often present with enlarged mediastinal lymph nodes on their chest computed tomography images. However, large irregular swollen lymph nodes are rarely reported in ACAP, and may therefore be confused with enlarged lymph node masses. In the present case report, the patient presented with lymph node masses, which were ameliorated to their normal size following antimicrobial treatment. The patient was 24 years old and otherwise healthy, which led to a pronounced and excessive immune response to pneumonia in the lymph nodes. Atypical pneumonia is difficult to diagnose based on imaging features. The present case report demonstrates that patients with pneumonia may present with unusually enlarged mediastinal lymph nodes, which are most likely, a result of a strong immune response to pneumonia.
\end{abstract}

\section{Introduction}

Adult community-acquired pneumonia (ACAP) is the most prevalent pulmonary infectious disease and is usually acquired via inhalation or aspiration of a pathogenic organism (1). Patients with ACAP may classically present with fever, a productive cough with purulent sputum, dyspnea and pleuritic chest pain. If a misdiagnosis is made, patients may become severely ill and may require a longer duration of therapy. ACAP is typically caused by Streptococcus pneumoniae, $\beta$-hemolytic Streptococci, Klebsiella pneumoniae or

Correspondence to: Dr Lanhua Zhang or Dr Jinming Xu, Department of Radiology, Yangpu Hospital, Tongji University School of Medicine, 450 Tengyue Road, Shanghai 200090, P.R. China E-mail: shzlanhua@163.com

E-mail: xu10611@hotmail.com

Abbreviations: ACAP, adult community-acquired pneumonia; CT, computed tomography; CAP, community-acquired pneumonia

Key words: community-acquired pneumonia, lymph node, tomography, X-ray computed tomography
Moraxella catarrhalis (2). General computed tomography (CT) features of patients with pneumonia of different etiologies include patchy opacity attenuation and ground-glass opacity shadowing of the lobe and segment (3). In addition, ACAP patients often present with enlarged mediastinal lymph nodes, visible in their chest CT images. However, giant irregular swollen lymph nodes associated with ACAP are rarely reported, and may be confused with enlarged lymph node masses. Such masses are frequently seen in patients with lung cancers, tuberculosis or lymphoma, and this may therefore lead to the misdiagnosis of ACAP as one of these conditions.

\section{Case report}

Written informed consent was provided by the patient regarding the publication of the case details in the present study. A 24-year old male was admitted to the emergency room of Yangpu Hospital (Shanghai, China) presenting with a moderate fever $\left(38.1-38.6^{\circ} \mathrm{C}\right)$, fatigue, mild cough with slight purulent yellow sputum, and intermittent shortness of breath. He had been suffering these symptoms for $\sim 3$ days. Prior to admission, he visited his local hospital because of similar symptoms, and a chest X-ray as performed. The X-ray images showed nothing remarkable. The patient then rested at home for 3 days, and took traditional Chinese medicine as prescribed (clearing heat and detoxification oral liquid; $10 \mathrm{ml}$ three times a day). However, there was no improvement to his cough and fever, and so he was referred to Yangpu Hospital. His past medical history indicated that he was healthy and had no major or serious illness except that he caught colds frequently. Clinical observations performed in the Emergency Department found that he exhibited a temperature of $38.6^{\circ} \mathrm{C}$, pulse rate of $92 \mathrm{bpm}$, respiratory rate of $28 \mathrm{breaths} / \mathrm{min}$, and blood pressure of $98 / 64 \mathrm{mmHg}$. The physical examination was unremarkable.

Blood was taken when the patient arrived in the emergency room. Laboratory blood tests indicated elevated C-reactive protein levels $(22 \mathrm{mg} / \mathrm{dl})$, normal hemoglobin with high leukocytosis (total leukocytes, $11,200 \mathrm{~mm}^{3}$; differential count, neutrophils $75.1 \%$, lymphocytes $20 \%$ ), a normal erythrocyte sedimentation rate of $16(0-20 \mathrm{~mm} / \mathrm{h})$, tuberculosis acid-fast staining, and L-Y culture negative. No sputum culture of bacterial pathogens was ordered. The chest $\mathrm{CT}$ scan revealed patchy areas of ground-glass attenuation and a small consolidation on the first segment of the right upper lung zone that 


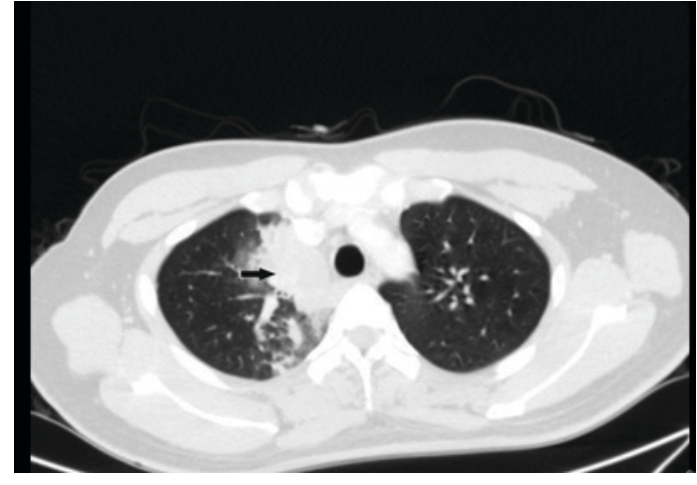

Figure 1. Small parenchymal opacification and patchy areas of ground-glass attenuation (black arrow) were presented in the right upper lung zones.

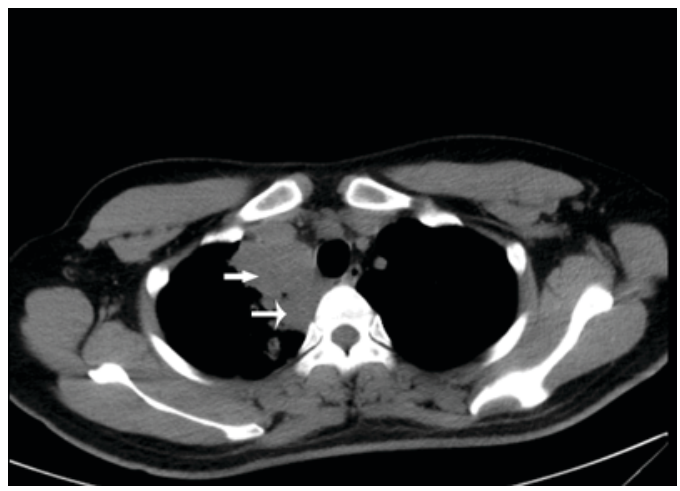

Figure 2. Lymphadenopathy mass (white arrows).

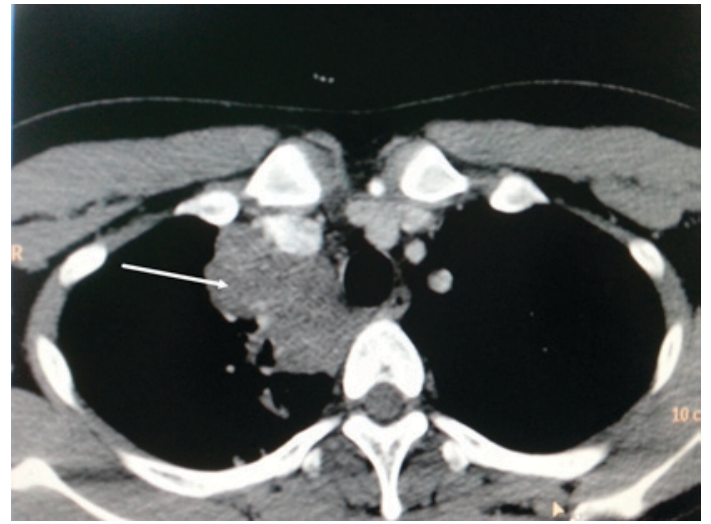

Figure 3. A giant irregular and mild enhanced mass (white arrow) was adjacent to the paratrachea on the contrast-enhanced computed tomography image.

was accompanied by a right upper mediastinal giant irregular lymphadenopathy mass (Figs. 1 and 2). Mild enhancement of the giant mediastinal lymphadenopathy mass was also noted on the contrast-enhanced CT scan (Fig. 3).

Radiological findings of pneumonia are characterized by the type and pattern of opacities, such as patchy areas of ground-glass attenuation and consolidation of zonal distribution (3). According to this, the patient was given a clinical diagnosis of bacterial pneumonia with lymphadenopathy and prescribed antibiotic treatment. The patient was administered with intravenous (IV) azithromycin ( $0.25 \mathrm{~g}$ drip, once daily

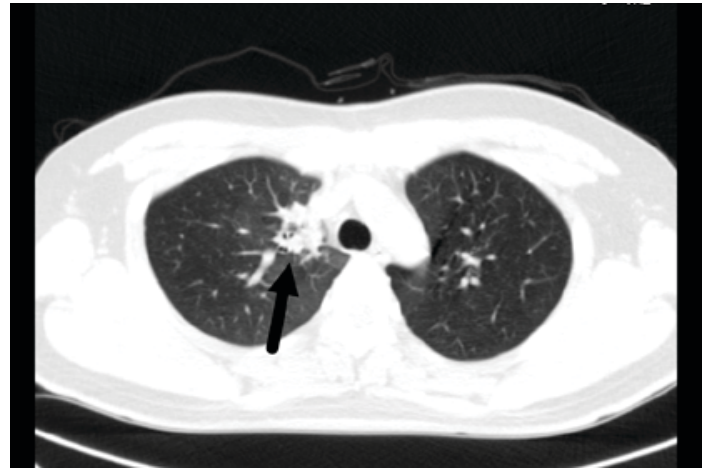

Figure 4. Following 7 days of antibiotic therapy, only a small nodular lesion (black arrow) was observed in the follow-up computed tomography scan image, and the irregular mass disappeared.

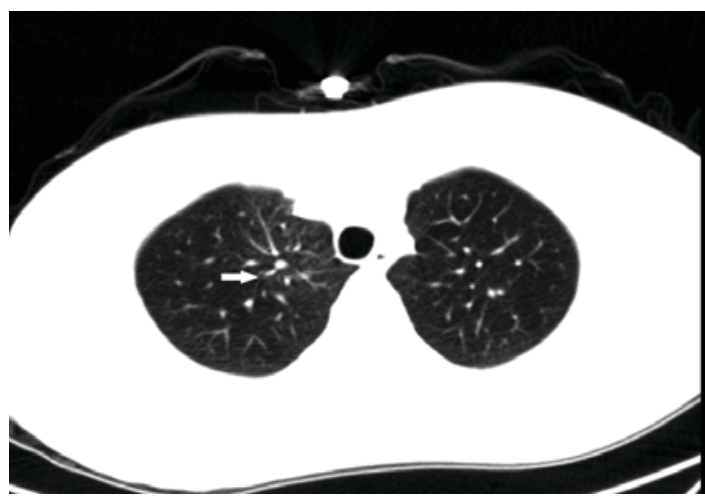

Figure 5. The initial abnormalities were completely resolved (white arrow) following 2 weeks of treatment based on the results of the computed tomography scan.

for 3 days), and subsequently was IV cefuroxime was administered ( $3 \mathrm{~g}$ drip, once daily for 10 days) as an outpatient. The patient was fully recovered following antibiotic therapy. All blood results were within the normal range, and a follow-up chest CT scan following 7 days of treatment revealed only a small nodular lesion (Fig. 4). Another CT scan was performed following 2 weeks of treatment and was found to be completely normal (Fig. 5).

The patient was eventually diagnosed with ACAP, non-severe type, based on his clinical manifestations, radiological findings, and the local epidemiological forecast.

\section{Discussion}

Community-acquired pneumonia (CAP) is acquired through normal social contact in the community as opposed to during hospitalization (hospital-acquired pneumonia) (1). CAP affects people of all ages and is a major cause of morbidity and health care expenditure $(4,5)$. It typically presents with problems such as difficulty breathing, fever, chest pains, and cough $(1,5)$. CAP may be divided into severe and non-severe types based on the pneumonia score (1).

The etiologies of CAP include bacteria, viruses, fungi, and parasites $(2,6)$. Historically, Streptococcus pneumoniae was the pathogen most commonly responsible for CAP (1). Prior to the advent of antibiotics and vaccination, it was a leading cause of mortality $(1,5)$. At present, the most common bacterial causes 


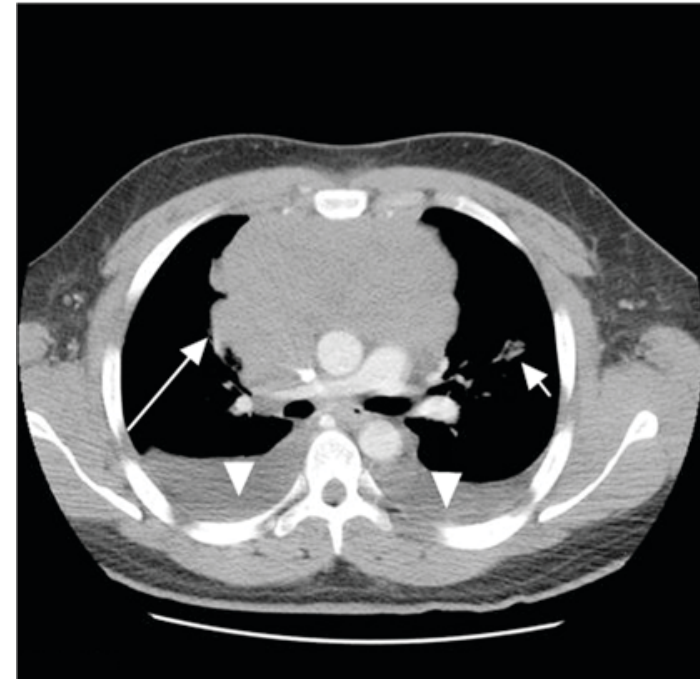

Figure 6. A computed tomography image of a patient with large B cell lymphoma. A large mediastinal mass with notable external compression of the superior vena cava (long white arrow) was noted, which was accompanied by moderate pericardial effusion. Large bilateral pleural effusions (white arrowheads) were also visible. A focal area of lung parenchymal consolidation was detected in the superior lingual segment of the left upper lobe (short white arrow).

of pneumonia are the so-called atypical bacteria, Mycoplasma pneumoniae and Chlamydophila pneumonia (7). Legionella pneumophila is also considered atypical; however, it is less common $(1,2,6)$. A survey was conducted in the Chinese metropolitan areas, including Shanghai and Beijing, where pneumonia is widespread, which found that Mycoplasma was the most common pathogen associated with pneumonia, followed by Streptococcus pneumonia (8). CAP may be diagnosed based on clinical manifestations alone, or using X-rays, sputum examinations, and other tests. In Yangpu Hospital, no routine bacterial culture is required for the diagnosis of mild CAP. The patient presented here fulfilled the non-severe CAP diagnosis criteria with the supporting evidence of clinical manifestations and chest X-ray imaging. He was safely treated as an outpatient.

Cases of pneumonia that are caused by different pathogens nonetheless typically have common presentations and features in chest X-rays and CT images $(3,9)$. CT images may reveal airspace involvement, which appears as lobular or segmental patchy areas of consolidation, nodular lesions, ground-glass opacity, and mild lymph node enlargement in isolated regions (3). Chest radiography is less accurate than $\mathrm{CT}$ in the detection of segmental and patchy areas of consolidation $(3,9)$.

In the present case study, the predominant radiographic abnormalities observed included the patchy areas of parenchymal opacification and ground-glass attenuation present in the right upper lung zones, and a large irregular mild enhanced mass mimicking lymphoma adjacent to the paratrachea in the right upper mediastinum on CT images. To the best of our knowledge, the enlarged lymph nodes may have resulted from infectious diseases, as the lymph nodes had a well-defined border and were apparently contrast-enhanced in the CT images, and the lymph nodes were scattered in mediastinal areas. Contrastingly, the lymph nodes in lymphoma may appear altogether in a large mass with irregular contour, and the CT images may demonstrate that the lymph nodes are mild-moderate contrast-enhanced with a border that is not well defined $(3,9)$. The follow-up CT scan performed following 2 weeks of treatment revealed that the initial abnormalities were resolved completely. To the best of our knowledge, it is extremely rare to observe pneumonia lesions that are accompanied by such a large mediastinal lymph node mass.

The pathogenic process of all types of pneumonia is able to cause acute or chronic inflammation in the mediastinal lymph nodes, lymph node hyperemia edema, hyperplasia of lymphocytes and macrophages, and infiltration of neutrophils, monocytes, and plasma cells $(7,10)$. In addition, the lymph nodes may experience necrosis and granuloma formation $(7,11)$.

Clinically, lymph node enlargement induced by acute inflammation is typically secondary to the corresponding drainage area of infection $(10,11)$. This case was atypical as abnormal lymph node enlargement with irregular fragments reaching $>40 \mathrm{~mm}$ were observed, most probably as a result of lung inflammation spreading to the adjacent mediastinal lymph nodes and causing excessive inflammatory reactions causing them to merge into a larger one. This patient was young, and his immune system was otherwise robust, which may be why he experienced such a strong and excessive response in the lymph nodes.

Unusually enlarged lymph nodes resulting from pneumonia may cause confusion in diagnosis. Typically, the inflammatory lymph nodes in pneumonia are characterized by mediastinal scattered lymph nodes forming a circular shadow, and the sizes are generally $\leq 15 \mathrm{~mm}$ (12). In the present case, there was a margin with a clear boundary, with no enhancement on the enhanced CT scan. The enlarged lymph nodes returned to their normal size with decreasing inflammation following effective antibiotic therapy.

In mediastinal lymph node tuberculosis, the lymph nodes are swollen and accompanied by central necrosis that frequently results in calcification in the healed sites $(3,10,13)$. In addition, ring-enhancement on the enhanced CT scan is observed (13).

In mediastinal lymphoma, the mediastinal lymphoma is typically located in the front mediastinum and is symmetrically and bilaterally widened with a wavy edge (seen more in Hodgkin's Disease) or one side is broadened (seen more in non-Hodgkin's lymphoma) (14). The lymph nodes are often fused into clumps, which results in mild-to-moderate enhancement on enhanced CT scans (Fig. 6) (15). The primary clinical symptoms include fever and superficial lymph node enlargement (14). The lymphoma may become smaller or disappear completely soon after radiation therapy (14-16).

In cases of lymph node metastasis, there is often a history of primary tumors, such as lung cancer (17). Additionally, enhanced CT scans of metastatic lymph nodes often have a notably enhanced ring or patch shapes $(16,18)$. As a result, it is difficult to make a differential diagnosis in the early metastatic stage only based on imaging.

In conclusion, mediastinal lymph node enlargement may be induced by many illnesses including metastasis, tuberculosis, chronic lymphocytic leukemia, Hodgkin's disease and more. Local lymph node enlargement typically indicates a local infection. Atypical pneumonia may cause confusion when making a diagnosis based on imaging analysis. The present 
case study demonstrates that unusually enlarged mediastinal lymph nodes may be present in patients with pneumonia and are most likely associated with a strong immune response to pneumonia.

\section{References}

1. Carbonara S, Monno L, Longo B and Angarano G: Community-acquired pneumonia. Curr Opin Pulm Med 15: 261-273, 2009.

2. Leesik H, Ani U, Juhani A and Altraja A: Microbial pathogens of adult community-acquired pneumonia in Southern Estonia. Medicina (Kaunas) 42: 384-394, 2006.

3. Nambu A, Ozawa K, Kobayashi N and Tago M: Imaging of community-acquired pneumonia: Roles of imaging examinations, imaging diagnosis of specific pathogens and discrimination from noninfectious diseases. World J Radiol 6: 779-793, 2014.

4. Broulette J, Yu H, Pyenson B, Iwasaki K and Sato R: The incidence rate and economic burden of community-acquired pneumonia in a working-age population. Am Health Drug Benefits 6: 494-503, 2013.

5. Herzig SJ, Howell MD, Ngo LH and Marcantonio ER: Acid-suppressive medication use and the risk for hospital-acquired pneumonia. JAMA 301: 2120-2128, 2009.

6. Kurutepe S, Ecemiş T, Ozgen A, Biçmen C, Celik P, Aktoğu Özkan $S$ and Sürücüoğlu S: Investigation of bacterial etiology with conventional and multiplex PCR methods in adult patients with community-acquired pneumonia. Mikrobiyol Bul 46: 523-531, 2012 (In Turkish).

7. Drasbek M, Nielsen PK, Persson K, Birkelund S and Christiansen G: Immune response to Mycoplasma pneumoniae P1 and P116 in patients with atypical pneumonia analyzed by ELISA. BMC Microbiol 4: 7, 2004.

8. Liu YN, Chen MJ, Zhao TM, Wang H, Wang R, Liu QF, Cai BQ, Cao B, Sun TY, Hu YJ, et al: A multicentre study on the pathogenic agents in 665 adult patients with community acquired pneumonia in cities of China. Zhonghua Jie He He Hu Xi Za Zhi 29: 3-8, 2006 (In Chinese).
9. Okada F, Ando Y, Tanoue S, Ishii R, Matsushita S, Ono A, Maeda T and Mori H: Radiological findings in acute Haemophilus influenzae pulmonary infection. Br J Radiol 85: 121-126, 2012.

10. Attili AK, Kazerooni EA, Gross BH, Flaherty KR and Martinez FJ: Thoracic lymph node enlargement in usual interstitial pneumonitis and nonspecific-interstitial pneumonitis: Prevalence, correlation with disease activity and temporal evolution. J Thorac Imaging 21: 288-292, 2006.

11. Weaver JL, Chapdelaine JM, Descotes J, Germolec D, Holsapple M, House R, Lebrec H, Meade J, Pieters R, Hastings KL and Dean JH: Evaluation of a lymph node proliferation assay for its ability to detect pharmaceuticals with potential to cause immune-mediated drug reactions. J Immunotoxicol 2: 11-20, 2005.

12. Qu JX, Gu L, Pu ZH, Yu XM, Liu YM, Li R, Wang YM, Cao B and Wang C; Beijing Network for Adult Community-Acquired Pneumonia (BNACAP): Viral etiology of community-acquired pneumonia among adolescents and adults with mild or moderate severity and its relation to age and severity. BMC Infect Dis 15: $89,2015$.

13. Hocke M, Menges M, Topalidis T, Dietrich CF and Stallmach A: Contrast-enhanced endoscopic ultrasound in discrimination between benign and malignant mediastinal and abdominal lymph nodes. J Cancer Res Clin Oncol 134: 473-480, 2008.

14. Sugimoto S, Soh J, Maki Y, Kurosaki T, Yamane M, Toyooka S, Oto T and Miyoshi S: Primary mediastinal lymphoma; a clinicopathologic case series. Kyobu Geka 65: 527-531, 2012 (In Japanese).

15. Dr Henry Knipe and Dr Frank Gaillard et al: Radiographic features, Mediastinal lymphoma

16. Mehrian P and Ebrahimzadeh SA: Differentiation between sarcoidosis and Hodgkin's lymphoma based on mediastinal lymph node involvement pattern: Evaluation using spiral CT scan. Pol J Radiol 78: 15-20, 2013.

17. Juanpere S, Cañete N, Ortuño P, Martínez S, Sanchez G and Bernado L: A diagnostic approach to the mediastinal masses. Insights Imaging 4: 29-52, 2013.

18. Tomiyama N, Honda O, Tsubamoto M, Inoue A, Sumikawa H, Kuriyama K, Kusumoto M, Johkoh T and Nakamura H: Anterior mediastinal tumors: Diagnostic accuracy of CT and MRI. Eur J Radiol 69: 280-288, 2009. 